\title{
A Confidence Framework for the Assessment of Optical Flow Performance
}

\author{
Patricia Márquez-Valle \\ Computer Science Department and Computer Vision Center, Edifici Q/O Campus UAB, Cerdanyola del Vallès, Barcelona, Spain \\ Advisor/s: Debora Gil and Aura Hernández-Sabaté \\ Date and location of PhD thesis defense: 27 July 2015, Universitat Autònoma de Barcelona
}

Received 29th June 2016; accepted 24th July 2016

\begin{abstract}
Optical Flow (OF) is the input of a wide range of decision support systems such as car driver assistance, UAV guiding or medical diagnose. In these real situations, the absence of ground truth forces to assess OF quality using quantities computed from either sequences or the computed optical flow itself. These quantities are generally known as Confidence Measures, CM. Even if we have a proper confidence measure we still need a way to evaluate its ability to discard pixels with an OF prone to have a large error. Current approaches only provide a descriptive evaluation of the CM performance but such approaches are not capable to fairly compare different confidence measures and optical flow algorithms. Thus, it is of prime importance to define a framework and a general road map for the evaluation of optical flow performance.

This thesis provides a framework able to decide which pairs "optical flow - confidence measure" (OF-CM) are best suited for optical flow error bounding given a confidence level determined by a decision support system. To design this framework we cover the following points:

- Descriptive scores. As a first step, we summarize and analyze the sources of inaccuracies in the output of optical flow algorithms. Second, we present several descriptive plots that visually assess CM capabilities for OF error bounding. In addition to the descriptive plots, given a plot representing OF-CM capabilities to bound the error, we provide a numeric score that categorizes the plot according to its decreasing profile, that is, a score assessing CM performance.

- Statistical framework. We provide a comparison framework that assesses the best suited OF-CM pair for error bounding that uses a two stage cascade process. First of all we assess the predictive value of the confidence measures by means of a descriptive plot. Then, for a sample of descriptive plots computed over training frames, we obtain a generic curve that will be used for sequences with no ground truth. As a second step, we evaluate the obtained general curve and its capabilities to really reflect the predictive value of a confidence measure using the variability across train frames by means of ANOVA.
\end{abstract}

The presented framework has shown its potential in the application on clinical decision support systems. In particular, we have analyzed the impact of the different image artifacts such as noise and decay to the output

Correspondence to: $\{$ aura,debora\}@cvc.uab.cat

Recommended for acceptance by David Vázquez Bérmudez

DOI http://dx.doi.org/10.5565/rev/elcvia.949

ELCVIA ISSN:1577-5097

Published by Computer Vision Center / Universitat Autònoma de Barcelona, Barcelona, Spain 

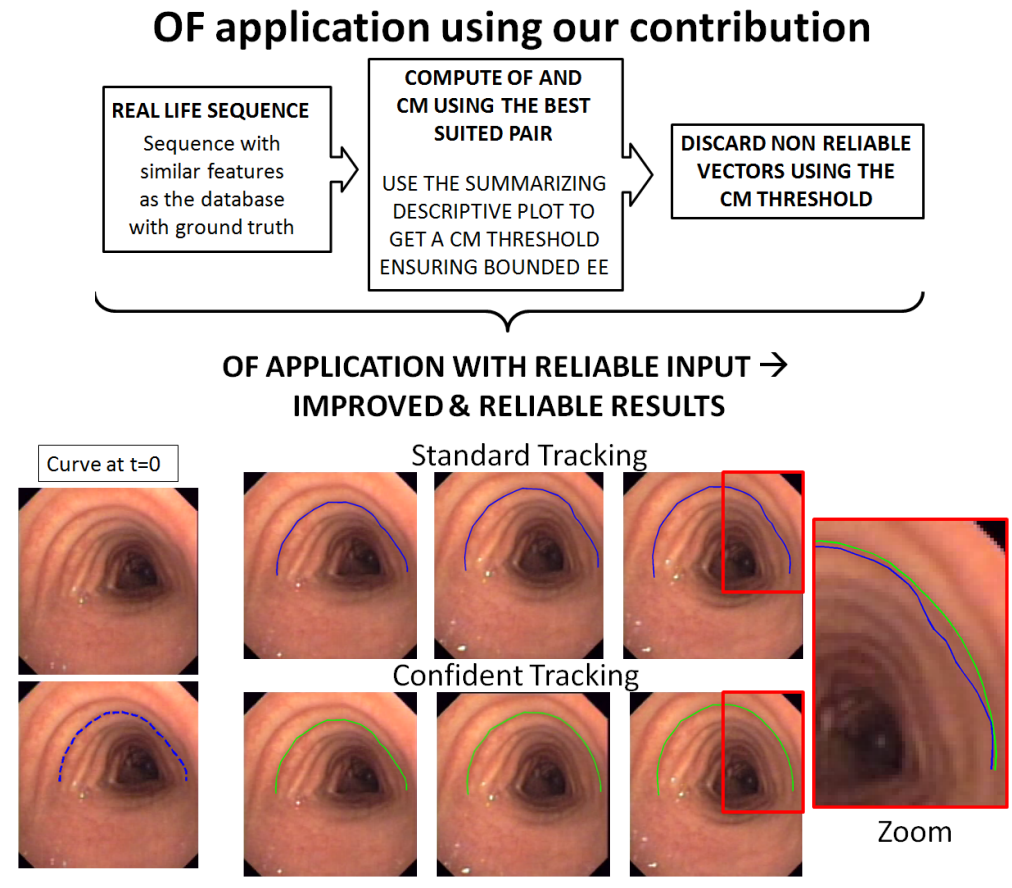

Figure 1: Application of the thesis contributions.

of optical flow in a cardiac diagnose system and we have improved the navigation inside the bronchial tree on bronchoscopy.

\section{Acknowledgment}

This work is supported by the Spanish MINECO projects TIN2012-33116, DPI2015- 65286- R, TIN201347913-C3-1-R, TRA2014-57088-C2-1-R and by the Secretaria d'Universitats i Recerca del Departament d'Economia i Coneixement de la Generalitat de Catalunya (2014-SGR-1470, and 2014-SGR-1506). Debora Gil is also Serra Hunter Fellow.

\section{References}

[1] H. Kause, P. Márquez-Valle, A. Fuster, A. Hernàndez-Sabaté, et al , "Quality Assessment of Optical Flow in Tagging MRI", Dutch Bio-Medical Engineering Conference, 2015.

[2] P. Márquez-Valle, H. Kause, A. Fuster, A. Hernàndez-Sabaté, et al , "Factors Affecting Optical Flow Performance in Tagging Magnetic Resonance Imaging", STACOM-MICCAI, pp. 231-238, 2014. doi:10.1007/978-3-319-14678-2_24.

[3] P. Márquez-Valle, D. Gil, R. Mester, A. Hernàndez-Sabaté, "Local Analysis of Confidence Measures for Optical Flow Quality Evaluation”, VISAPP, pp. 450-457, 2014.

[4] P. Márquez-Valle, D. Gil, A. Hernàndez-Sabaté, "Evaluation of the Capabilities of Confidence Measures for Assessing Optical Flow Quality”, IEEE ICCV - Workshops, pp. 624-631, 2013. doi:10.1109/iccvw.2013.86.

[5] P. Márquez-Valle, D. Gil, A. Hernàndez-Sabaté, Daniel Kondermann, "When Is a Confidence Measure Good Enough?”, ICVS, pp. 344-353, 2013. doi:10.1007/978-3-642-39402-7_35. 
[6] P. Márquez-Valle, D. Gil, A. Hernàndez-Sabaté, "A Complete Confidence Framework for Optical Flow", ECCV - Workshops, pp. 124-133, 2012.

[7] P. Márquez-Valle, D. Gil, A. Hernàndez-Sabaté, "Error Analysis for Lucas-Kanade Based Schemes", ICIAR, pp. 184-191, 2012. doi:10.1007/978-3-642-31295-3_22.

[8] P. Márquez-Valle, D. Gil, A. Hernàndez-Sabaté, ”A confidence measure for assessing optical flow accuracy in the absence of ground truth", ICCV - Workshops, pp. 2042-2049, 2011. 